\title{
Política de estímulo à iniciação científica: impacto no coeficiente de rendimento de graduandos em Odontologia
}

\author{
Elisa Fonseca Nardini*; Cecilia Pedroso Turssi**; Almenara de Souza Fonseca Silva**; Flávia Martão \\ Flório** \\ * Estudante, Curso de Odontologia, Faculdade São Leopoldo \\ Mandic \\ ** Docente, Curso de Odontologia, Faculdade São Leopoldo \\ Mandic
}

Recebido em 27/02/2018. Aprovado em 07/01/2019.

\begin{abstract}
RESUMO
O presente estudo observacional do tipo caso-controle avaliou o impacto da participação no Programa de Iniciação Científica (PIC) no coeficiente de rendimento (CR) acadêmico de graduandos em Odontologia. Alunos de uma instituição de ensino superior (IES) particular localizada em Campinas (SP) foram divididos em dois grupos: caso (GCs; vinculados ao PIC 2013 a 2016) e controle (GCt estudantes matriculados e/ou egressos que se enquadraram aos critérios de inclusão). Calculou-se o CR dos participantes, ponderado pela carga horária das disciplinas, sendo para o GCs: nos semestres anteriores à participação no PIC e nos semestres letivos cursados em concomitância ou posteriormente à $1^{\mathrm{a}}$ participação no programa. Para o $\mathrm{GCt}$, considerou-se o CR dos semestres cursados. Vincularam-se ao PIC 58 estudantes, sendo 15,5\% (9) na $1^{\text {a }}$ edição (2013-2014); 27,6\% (16) na $2^{\text {a }}$ (2014-2015); 29,3\% (17) na $3^{\mathrm{a}}$ (2015-2016) e 27,6\% (16) na $4^{\mathrm{a}}$ edição (2016-2017). Dentre os aptos a participar do GCt, sorteou-se 60 estudantes. Verificou-se que houve um aumento significativo ( $\mathrm{p}<0,0001$, teste $\mathrm{t}$ pareado) nos CRs calculados para GCs antes do PIC $(7,73 \pm 0,49)$ e depois do PIC $(7,89 \pm 0,46)$. Estudantes GCs tiveram CR mais alto do que aqueles do GCt $(7,45 \pm 0,56)$, tanto previamente quanto após sua participação no PIC ( $p<0,0001$, teste t para uma média). Concluise que o estímulo à iniciação científica por meio da institucionalização de programas acadêmicos diferencia o rendimento escolar dos participantes.

Descritores: Avaliação Educacional. Pesquisa em Odontologia. Estudante de Odontologia.
\end{abstract}

\section{INTRODUÇÃO}

O desafio contemporâneo das universidades é formar indivíduos que, além de buscar, sejam capazes de utilizar racionalmente os conhecimentos e, neste contexto, as atividades voltadas para a solução de problemas tornam-se importantes instrumentos na formação dos estudantes ${ }^{1}$. Uma formação acadêmica completa tem como requisitos além das atividades de ensino e extensão, a concomitante participação em atividades de pesquisa ${ }^{2}$, diretamente vinculadas à geração e ao domínio do conhecimento ${ }^{3}$ e à 
possibilidade de direcionamento à uma carreira envolvendo pesquisa, seja no meio acadêmico, seja no setor produtivo ${ }^{4}$.

Dentro dessa perspectiva, é notório o conhecimento de que a inserção precoce do graduando na iniciação científica (IC) é um instrumento valioso para aprimorar qualidades desejadas em um profissional de nível superior ${ }^{5}$, bem como para estimular e despertar a vocação para a pesquisa ${ }^{3,6,7}$. Com isso, possibilita-se um direcionamento mais precoce de estudantes para a pós-graduação Stricto $\operatorname{Sensu}^{8,9}$ com menores taxas de evasão ${ }^{4}$.

De forma geral, é inegável que a iniciação científica resulta numa formação diferenciada já que são exigidas competências que normalmente não são trabalhadas em sala de aula; além do fato de que, para a participação nos programas institucionais, os estudantes devem ter e manter um rendimento escolar satisfatório. Silva e Martins $(2014)^{10}$ confirmaram tal premissa para estudantes de Química de uma universidade federal, com base na avaliação dos históricos escolares e realização de entrevistas com estudantes bolsistas e exbolsistas do PIC.

A pesquisa científica proporciona ao estudante maior integração com a instituição e com os conteúdos, culminando em melhor desempenho acadêmico e formação mais abrangente. Souza e Marques $(2011)^{11}$, com base na percepção dos graduandos, comprovaram essa relação já que a maioria dos estudantes de Química participantes da IC afirmaram ter obtido aumento significativo no rendimento acadêmico. Este achado foi também verificado em outros estudos que buscaram a percepção dos estudantes das quatro áreas de ensino e pesquisa da UNICAMP: artes, humanas, biológicas e exatas, quanto aos benefícios da $\mathrm{IC}^{12} \mathrm{e}$ também indicaram melhores habilidades para atuar no cenário atual do mercado de trabalho ${ }^{13}$.

Dentre as instituições privadas, historicamente são menos frequentes as decisões estratégicas voltadas ao estímulo e desenvolvimento de pesquisas científicas, sendo verificada uma grande diferença nesta atividade a favor das instituições fomentadas pelo poder público ${ }^{3}$. Dados um pouco mais recentes apontam que as instituições privadas foram responsáveis por menos de $20 \%$ do total de trabalhos apresentados em uma Reunião Anual da Sociedade Brasileira de Pesquisa Odontológica $(\mathrm{SBPqO})^{14}$. Massi e Queiroz $(2010)^{5}$ indicam que pesquisas sobre a atividade de IC realizadas no país eram incipientes e, em complemento, para estudantes de Odontologia, o impacto da IC no rendimento acadêmico não foi anteriormente avaliado considerando a mensuração do proposto indicador acadêmico.

É dentro dessa perspectiva de necessidade de atualização do conhecimento disponível, que o presente estudo teve como objetivo avaliar o impacto da participação no PIC no coeficiente de rendimento acadêmico (CR) de graduandos em Odontologia.

\section{METODOLOGIA}

Este estudo observacional do tipo casocontrole foi conduzido de acordo com os preceitos determinados pela Resolução 422 de 12/2012 do Conselho Nacional de Saúde do Ministério da Saúde, e aprovado pelo Comitê de Ética em Pesquisa, segundo CAAE 61651516.1.0000.5374.

O PIC da Faculdade São Leopoldo Mandic foi institucionalizado em 2013 e considerou-se para o presente estudo as turmas que puderam se inscrever no programa em suas quatro primeiras edições. Os nomes dos estudantes inscritos foram obtidos por intermédio da secretaria do programa, sendo 9 participantes da $1^{\mathrm{a}}$ edição (2013-2014); 16 na $2^{a}$ edição (2014-2015); 17 na $3^{\text {a }}$ edição (20152016) e 16 na $4^{\text {a }}$ edição (2016-2017). Considerouse para a composição e coleta de dados do grupo caso (GCs), a primeira participação do aluno no PIC, resultando em um grupo composto por 58 estudantes. 
O grupo controle (GCt) foi selecionado com base na listagem dos 147 estudantes matriculados no curso de Odontologia e que eram aptos a participar dos processos seletivos para as referidas edições do PIC. Foram excluídos 91 deles que participaram, como autores de trabalho, do Seminário de Iniciação Científica em qualquer uma das edições de 2014 a 2017, resultando em 89 estudantes aptos a compor o grupo controle. Deste total, por meio de tabela de números aleatórios, sorteou-se os estudantes, de forma que a proporção entre os grupos fosse mantida em 1:1.

A análise documental consistiu na apuração do CR, calculado pela média ponderada das notas obtidas nas disciplinas, considerando como peso as suas respectivas cargas horárias. Para o grupo caso, calculou-se o CR das disciplinas cursadas previamente e após participação no PIC. Quando houve participação em mais de uma edição do PIC, foi considerado como ponto de corte para o cálculo dos CRs, o período da primeira vinculação ao PIC.
Para os estudantes do grupo controle, utilizou-se o CR médio de todos os semestres cursados e finalizados até a coleta de dados.

Os dados foram apurados e quantificados em frequências absolutas e relativas. Para investigar se houve diferença nos CRs no grupo caso antes e após o PIC, foi empregado o teste t pareado. Para comparação dos CRs antes e após o PIC com o CR calculado para o grupo controle, utilizou-se o teste t para uma média. O nível de significância adotado foi de $5 \%$ e os testes foram realizados no programa Bioestat 5.0

\section{RESULTADOS}

Na tabela 1 constam os CRs calculados para o GCs antes e após a participação no PIC, com aumento significativo $(\mathrm{p}<0,0001)$.

Em comparação com o CR dos estudantes que compuseram o GCt $(7,45 \pm 0,56)$, nota-se que o valor médio do rendimento foi sempre maior no GCs (tabela 2).

Tabela 1. Grupo caso: Análise exploratória dos coeficientes de rendimento antes e depois do ingresso no PIC

\begin{tabular}{cccccc}
\hline & Média & DP & Mínimo & Máximo & Mediana \\
\hline CR antes & $7,73 \mathrm{a}$ & 0,49 & 6,77 & 8,80 & 7,64 \\
CR depois & $7,89 \mathrm{~b}$ & 0,46 & 7,03 & 8,89 & 7,83 \\
\hline \multicolumn{4}{l}{ Médias seguidas por letras distintas diferem entre si pelo teste t de Student pareado }
\end{tabular}

Tabela 2. Comparação do CRs entre os grupos caso e controle

\begin{tabular}{clc}
\hline \multicolumn{1}{c}{ Grupo } & Coeficiente de Rendimento \\
\hline Caso & Antes PIC & $7,73^{*}$ \\
& Depois PIC & $7,89 *$ \\
\hline Controle & Média & 7,45 \\
& DP & 0,56 \\
& Mínimo & 6,10 \\
& Máximo & 8,21 \\
& Mediana & 0,56 \\
\hline \multirow{2}{*}{ Médias caso marcadas com $*$ diferem do controle $(\mathrm{p}<0,0001 ;$ Teste t para uma amostra $)$}
\end{tabular}




\section{DISCUSSÃO}

A realização da IC durante a graduação contribui para a formação de um bom profissional e deve ser valorizada pelas instituições de ensino superior (IES) favorecendo uma completa e crítica formação profissional ${ }^{2,5,10}$. Verificou-se no presente estudo que os estudantes inscritos no PIC apresentaram um aumento do rendimento acadêmico, tanto em relação ao desempenho individual, que melhorou após o programa, como também, em relação aos estudantes do grupo controle, cujo CR médio foi sempre menor do que $\mathrm{o}$ verificado para o grupo caso. Este fato reforça a fala de Chisini et al. $(2017)^{2}$ de que a possibilidade de ingressar em programas de IC é uma das principais oportunidades que as IES podem oferecer aos seus estudantes para integração ao ambiente acadêmico e leva à premissa de que a criação de programas institucionais de fato oferece um diferencial acadêmico.

A literatura consultada não apresenta dados recentes sobre a realidade da produção científica em função da categoria da instituição, tendo-se relatos de que essa é uma realidade mais comum nas instituições públicas ${ }^{3}$, porém o Relatório do Centro de Gestão e Estudos Estratégicos aponta que há instituições privadas focadas e empenhadas nessa vertente ${ }^{4}$.

$\mathrm{Na}$ IES do presente estudo, o PIC foi institucionalizado em 2013 com o objetivo despertar a vocação científica e incentivar novos talentos entre estudantes de graduação, tendo como parte essencial de sua missão o incentivo à pesquisa técnico-científica. Nesta linha, além das 4 quotas institucionais anuais do Programa Institucional de Bolsas de Iniciação Científica do Conselho Nacional de Desenvolvimento Científico e Tecnológico (PIBIC/CNPq), a instituição fornece em contrapartida outras seis bolsas institucionais, de forma que mais estudantes possam ser contemplados com este auxílio financeiro. Além disso, embora o curso de graduação seja relativamente recente - primeira turma iniciada em 2004 - o constante estímulo à iniciação científica contribuiu para a captação de fomento de órgãos públicos como a Fundação de Amparo à Pesquisa do Estado de São Paulo (FAPESP), assim como oriundos da iniciativa privada.

$\mathrm{Na}$ lógica discutida por Pires (2009) ${ }^{7}$, em 2016 foi lançado um edital específico para o acompanhamento de ingressantes e egressos do PIC, que contempla mais uma quota institucional extra, destinada à avaliação do programa, estimulando dessa forma que pesquisadores realizem o acompanhamento da prática profissional dos ingressantes e egressos do PIC. A presente pesquisa foi contemplada no $1^{\circ}$ edital e o perfil traçado por esse e outros estudos desta linha permitirão que o programa seja aperfeiçoado e valorizado.

A IC constitui-se num programa cujo principal objetivo é iniciar graduandos no universo da pesquisa científica ${ }^{1}$. Esse novo contexto, requer habilidades não trabalhadas em sala de aula, como testar técnicas e hipóteses, buscar bibliografias e desenvolver metodologias, de forma que o resultado final desse processo culmine em um aumento do conhecimento e desempenho escolar, uma vez que a necessidade de se obter conhecimento além da sala de aula e adquirir capacidade de gestão de tempo e autonomia têm influência direta no processo de aprendizagem ${ }^{15,16}$. O aumento do CR dos estudantes verificado após a vinculação ao programa pode ser reflexo do desenvolvimento e valoração destas habilidades.

Em contraponto, Silva e Martins (2014) ${ }^{10}$, considerando flutuações nos rendimentos individuais observadas no período inicial da vigência do programa de IC de uma IES federal, discutem aspectos relacionados à dificuldade dos estudantes em conciliar os estudos com a fase de adaptação às atividades decorrentes das novas responsabilidades demandadas pela IC. Estudos posteriores devem considerar aspectos individuais 
dos estudantes que participam do PIC, já que dificuldades no contexto universitário são de diversas naturezas, passando tanto pelas questões individuais dos estudantes como também pelas novas exigências acadêmicas e o novo ambiente, influenciando o desempenho e o desenvolvimento psicossocial dos estudantes ${ }^{17}$.

Neste contexto, o ingresso na vida acadêmica marca o início de uma etapa repleta de expectativas, de novas responsabilidades, de compromissos e da construção de uma nova identidade $^{18}$. De acordo com as atividades exercidas no curso, o ambiente acadêmico influencia diferentemente cada aluno ${ }^{19}$ e a assimilação deste novo universo requer múltiplas adaptações que são influenciadas por características próprias do aluno, pelas oportunidades que a instituição oferece e pelo desempenho acadêmico apresentado por cada estudante $\mathrm{e}^{20,21}$

De todo modo, a melhoria do CR verificada no presente estudo pode também ser compreendida, pela IES do estudo ser uma instituição privada, que não dispõe de programas sociais de bolsas ou financiamentos públicos. Os estudantes matriculados em seus cursos são classificados, segundo Brito et al. (2008) $)^{22}$ como do tipo "clássico", por pertencerem a um segmento privilegiado da sociedade que busca a manutenção ou melhoria de sua condição social e que dispõe de tempo para estudar, tem idade, formação intelectual que transcende os conteúdos escolares, financiamento familiar, disposição para atividades diversificadas social e culturalmente, características estas que coadunam com as exigências acadêmicas.

No contexto da presente amostra, os impactos verificados ainda são a curto prazo, sendo interessante investigar, em estudos futuros, os impactos da IC em outros domínios e indicadores relacionados às decisões profissionais que se seguem à graduação.

\section{CONCLUSÃO}

O estímulo à iniciação científica por meio da institucionalização de programas contribuiu para a melhoria do rendimento acadêmico dos estudantes de Odontologia.

\section{ABSTRACT \\ Impact of a research mentorship program on the performance coefficient of Dentistry students}

This observational, case-control type study evaluated the impact of participation in a Research Mentorship Program (RMP) on the academic performance coefficient (PC) of undergraduates of a dentistry course. Students from a private higher education institution (HEI) located in Campinas (SP) were divided into two groups: case (CGs; who participated in the RMP between 2013 and 2016) and control (CTGs, enrolled students and/or graduates who met the inclusion criteria). The PC of the participants was calculated, considering the study hours of the subjects. Those of the CGs were based on the semesters prior to participation in the RMP and those taught concurrently or after first participation in the program. For the CTGs all semesters studied were considered. A total of 58 students were linked to the RMP, with $15.5 \%$ (9) in the 1st edition (2013-2014); 27.6\% (16) in the 2nd (2014-2015); 29.3\% (17) in the 3rd (20152016) and $27.6 \%$ (16) in the 4th edition (20162017). A total of 60 students were randomly selected for inclusion in the CTG. It was found that there was a significant increase in the calculated PCs of the CGs $(\mathrm{p}<0.0001$, paired $\mathrm{t}$ test) from before $(7.73 \pm 0.49)$ to after the RMP $(7.89 \pm 0.46)$. CG students had a higher PC than students of the CTG $(7.45 \pm 0.56)$, both before and after their participation in the RMP $(p<0.0001$, one sample t-test $)$ ). It can be concluded that research mentorship stimulus through the establishing of academic programs makes a difference to the scholastic performance of the participants.

Descriptors: Educational Assessment. Research in Dentistry. Dentistry Students. 


\section{REFERÊNCIAS}

1. Bastos F, Martins F, Alves M, Terra M, Lemos CS. A Importância da Iniciação Científica para os alunos de graduação em Biomedicina. Novo Enfoque. 2010;11 (11):61-6.

2. Chisini LA, Silva HG, Nóbrega KHS, Conde MCM, Corrêa MB, Demarco FF. Análise descritiva dos trabalhos de conclusão de curso da Faculdade de Odontologia. Rev ABENO. 2017;17(1):8-15.

3. Fava de Morais F, Fava M. A iniciação científica: muitas vantagens e poucos riscos. São Paulo em Perspectiva. 2000; 14(1):73-7.

4. Centro de Gestão e Estudos EstratégicosCGEE. A Formação de novos quadros para CT\&I: avaliação do programa institucional de bolsas de iniciação científica (Pibic). Brasília, DF: 2017. 44p.

5. Massi LQ, Queiroz SLL. Estudos sobre iniciação científica no Brasil: uma revisão. Rev Eletr Cad Pesqui. 2010;40(139):173-97.

6. Humphrey P, Woods NF. Involving Undergraduate Students in Faculty Research. J Nurs Educ.1980;19(5):4-6.

7. Pires RCM. Formação inicial do professor pesquisador através do programa PIBIC/CNPq: o que nos diz a prática profissional de egressos? Avaliação (Campinas). 2009;14(2), 487-514.

8. Nogueira MA, Canaan MG. Os "iniciados": os bolsistas de iniciação científica e suas trajetórias acadêmicas. Rev TOMO. 2009; 15(42):41-70.

9. Bardagi MP, Hutz CS. Mercado de trabalho, desempenho acadêmico e o impacto sobre a satisfação universitária. Ciên Hum. 2012; 46(1):183-98.

10. Silva MGL, Martins AFP. Reflexões do PIBID - Química da UFRN: Para Além da Iniciação à Docência. Quím Nova Esc. 2014; 36(2):101-107.
11. Sousa JMT, Marques HVCF. Contribuições da iniciação científica no processo de ensino e aprendizagem de química. Cad Pesqui. 2011;18(3):31-39.

12. Bridi JCA. A Iniciação científica na formação do universitário. 2004. Dissertação (Mestrado em Educação) Faculdade de Educação, Universidade Estadual de Campinas, Campinas

13. Cunha MR, Fonsêca GS, de Araujo ME, Zilbovicius C. A Iniciação Científica como estratégia pedagógica para integrar ensino e serviço no SUS. Rev ABENO. 2016;16(2):33-44

14. Cavalcanti AL, Borja de Melo TRN, Barroso KMA, Cunha de Souza FE, Maia AMA, Silva ALO. Perfil da Pesquisa Científica em Odontologia Realizada no Brasil. Pesq Bras Odontoped Clin Integr. 2004. 4(2): 99-104.

15. Machineski RS, Machado ACTA, Silva RTM. A importância do estágio e do programa de iniciação científica na formação profissional e científica. Enciclop Biosf. 2011;7(13):1562-74.

16. Santos AAA, Mognon, JF, Lima TH, Cunha NB. A relação entre vida acadêmica e a motivação para aprender em universitários. Psicol Esc Educ. 2011;15(2):283-90.

17. Cunha SM, Carrillo DM. O processo de adaptação ao ensino superior e o rendimento acadêmico. Psicol Escolar Educ. 2005. 9(2): 215-24.

18. Fernandes VMP. Adaptação acadêmica e autoeficácia em estudantes universitários do $1^{\circ}$ ciclo de estudos [Dissertação de Mestrado]. Porto: Faculdade de Ciências Humanas e Sociais da Universidade Fernando Pessoa, 2011.

19. Santos L, Almeida LS. Vivências acadêmicas e rendimento escolar: estudo com alunos universitários do $1 .^{\circ}$ ano. Análise Psicológica. 2001; 19(2):205-17 
20. Igue EA, Bariani ICD, Milanesi PVB. Vivência acadêmica e expectativas de universitários ingressantes e concluintes. Psico-USF. 2008; 13(2) 155-64.

21. Basso C. Vivências acadêmicas e os fatores da permanência/evasão de estudantes no ensino superior. In: I Seminário Iberoamericano: as transições dos estudantes, um desafio para as universidades, 2013, Itajaí. I Seminário Iberoamericano: as transições dos estudantes, um desfaio para as universidades, 2013.
22. Britto LPL, Silva EO, Castilho KC, Abreu TM. Conhecimento e formação nas IES periféricas perfil do aluno "novo" da educação superior. Avaliação. 2008;13(3): 777-91.

\section{Correspondência para:}

Flávia Martão Flório

e-mail: flavia.florio@slmandic.edu.br

Rua José Rocha Junqueira, 13

13045-755 Campinas/SP 